\title{
GT2018-76826
}

\section{NUMERICAL AND EXPERIMENTAL INVESTIGATIONS ON OPTIMIZED 3D COMPRESSOR AIRFOILS}

\author{
Jan Mihalyovics* \\ Christian Brück \\ Dieter Peitsch \\ Chair for Aero Engines \\ Institute of Aeronautics and Astronautics \\ Technische Universität Berlin \\ Marchstr. 12-14 \\ 10587 Berlin, Germany \\ Email: Jan.Mihalyovics@tu-berlin.de
}

\author{
Ilias Vasilopoulos \\ Marcus Meyer \\ Rolls-Royce Deutschland Ltd \& Co KG \\ Eschenweg 11 \\ 15827 Blankenfelde-Mahlow, Germany \\ Email: Ilias.Vasilopoulos@rolls-royce.com
}

\section{ABSTRACT}

The objective of the presented work is to perform numerical and experimental studies on compressor stators. This paper presents the modification of a baseline stator design using numerical optimization resulting in a new $3 D$ stator. The Rolls Royce in-house compressible flow solver HYDRA was employed to predict the $3 D$ flow, solving the steady RANS equations with the Spalart-Allmaras turbulence model, and its corresponding discrete adjoint solver. The performance gradients with respect to the input design parameters were used to optimize the stator blade with respect to the total pressure loss over a prescribed incidence range, while additionally minimizing the flow deviation from the axial direction at the stator exit. Non-uniform profile boundary conditions, being derived from the experimental measurements, have been defined at the inlet of the CFD domain. The presented results show a remarkable decrease in the axial exit flow angle deviation and a minor decrease in the total pressure loss. Experiments were conducted on two compressor blade sets investigating the three-dimensional flow in an annular compressor stator cascade. Comparing the baseline flow of the $42^{\circ}$ turning stator shows that the optimized stator design minimizes the secondary flow phenomena. The experimental investigation dis-

${ }^{*}$ Address all correspondence to this author. cusses the impact of steady flow conditions on each stator design while focusing on the comparison of the $3 D$ optimized design to the baseline case. The flow conditions were investigated using five-hole probe pressure measurements in the wake of the blades. Furthermore, oil-flow visualization was applied to characterize flow phenomena. These experimental results are compared with the CFD calculations.

\section{INTRODUCTION}

Reducing greenhouse gas emissions, noise and specific fuel consumption in aviation has already been a key factor for some time. Especially in gas turbine engineering and turbomachinery design, the efficiency may not be reduced due to new environmental regulations but rather need to be enhanced by disruptive new ideas, introducing new technological challenges. One approach is to pass from a constant pressure combustion to a constant volume combustion [1,2]. A thermodynamic process describing such a combustion is the so-called pulse detonation engine $(P D E)$ detonating the ignited fuel under near isochoric conditions. The periodic occurrence of shock waves at the outlet and blockage at the inlet of the combustion tubes lead to unsteady flow conditions upstream and downstream of the combustion section. Thus, the compressor suffers from non-steady outlet

Copyright @ $\odot 2018$ by Rolls-Royce Deutschland Ldt. \& Co KG 
conditions which are imposed by the PDE combustion tubes. As a major consequence, this leads to a variation of the incidence angle at the leading edge of the last compressor stator and the occurrence of unsteady flow characteristics. These have a negative influence on the overall aerodynamic response and the stable operation of the compressor and need to be addressed [3]. Corner vortices and possible corner separation may form in between a blade's suction side and the hub and/or casing-wall as shown by Beselt et.al. [4]. The highest losses occur within these regions [5,6]. Gbadebo [7] showed that a variation in incidence angle led to changes in size and characteristics of those vortices. As the pitch-to-chord ratio in the annular test rig is smaller on the hub side than at the tip, an asymmetrical occurrence of the corner vortices is observed as shown by Brück [8]. Utilizing different means of flow control has shown that the blade passage losses can be successfully reduced [9]. Intensive studies have been conducted on handling the flow of the suction side, which is largely influenced by unsteady inflow conditions. Active methods have been investigated as shown in [10-14]. Opposed to active flow control, relying on additional energy to influence the flow, the passive approach is mostly driven by changing pressure gradients. Investigations in enhanced mixing by means of vortex generators between main flow and decelerated boundary layer have been made by Hergt [15]. The aim of the present investigation is to reduce the losses caused by the corner separation in terms of passive flow control using geometric optimization, regarding a fixed inflow condition with focus on robustness against changes of the inlet flow angle $\alpha_{I}$. In particular, this study presents a 3D optimization using a very rich design space of a stator cascade which is prone to 3D separated flows, improving the passage characteristics significantly in hindsight of future studies to be conducted under largely unsteady compressor conditions.

\section{NUMERICAL SETUP AND CFD SIMULATION}

Optimization methods are divided into two main categories: gradient-free and gradient-based methods. Gradient-free approaches such as evolutionary algorithms are global optimizers which guarantee finding a global optimum solution to a problem but require a large number of function evaluations. The computational cost of these techniques scale with the number of design parameters, but can be mitigated using response surface approximations as demonstrated in $[16,17]$. In gradient-based methods the optimization is driven by the gradient information to more efficiently minimize the problem's cost function. Their main drawback is that they are likely to be trapped in local optima.

In Computational Fluid Dynamics $(C F D)$ applications the gradient of the objective function with respect to the design parameters can be computed using finite-differences. A much more efficient way to calculate the gradient information is using the adjoint method presented in [18-20], thus providing the full gradient at a cost of solving a linear system. Although the adjoint technique can be robust and stable for realistic flow characteristics, such as flow separations [21], challenges related to its industrial applicability are not sufficiently resolved yet. Research towards that direction is currently being conducted within the EC ITN IODA project [22].

In order to perform gradient-based optimization using a CADbased parameterization, the sensitivities of the model's boundary with respect to the design parameters have to be computed. In this work, the geometric sensitivities are calculated using finitedifferences inside Parablading [23], the Rolls-Royce in-house aerofoil design software. Alternatively, an approach using design velocity is presented in [24]. This section describes the numerical setup with focus on the underlying steps that need to be considered in order to perform the optimization and subsequent $C F D$ simulations. The same setup has been previously used in [25], where more details can be found.

\section{Test Case Description}

The initial geometry of the TurboLab Stator can be found in [26]. The goal is to optimize a baseline stator $(B S)$ blade with respect to two optimization criteria, which leads to a multiobjective optimization problem. The first objective to be minimized is the total pressure loss between the inlet and the outlet section defined by the total pressure loss coefficient:

$$
\omega=\left(\frac{p_{t, I}-p_{t, E}}{p_{t, I}-p_{I}}\right),
$$

where $p$ and $p_{t}$ are computed using mass-averaging over the corresponding cross section. The second objective is the flow angle deviation from the axial direction $x$ at the stator outlet $\alpha_{E}$, which takes into account both the circumferential and radial components. These two objectives are contradicting, because the higher turning — which is required in this case to achieve a more axial outflow - produces more losses. Moreover, in order to guarantee a robust optimum blade against inlet angle variations, a multi-point optimization problem is defined. The design point $(D P)$ inlet whirl angle's non-uniform profile (shown in Fig. 9) is shifted by $i= \pm 5^{\circ}$, thus two off-design operating points are considered $(O D+$ and $O D-)$. The prescribed weights for the different operating conditions to be considered by the optimization technique are $50 \%$ for the $D P$ and $25 \%$ for each $O D$ point. Various manufacturing constraints (such as LE and TE radii, axial chord length, hub and tip assembly holes) have to be taken into account during the optimization. A detailed description of their definition can be found in [26]. This document also includes information about the boundary conditions of the case. The only deviation in this work is the inclusion of non-uniform inlet boundary conditions matching the test rig measurements. Finally, the mass flow of the full annulus is to be kept at $9.5 \pm 0.1 \mathrm{~kg} / \mathrm{s}$ over the whole operating range.

Copyright (C) 2018 by Rolls-Royce Deutschland Ldt. \& Co KG 

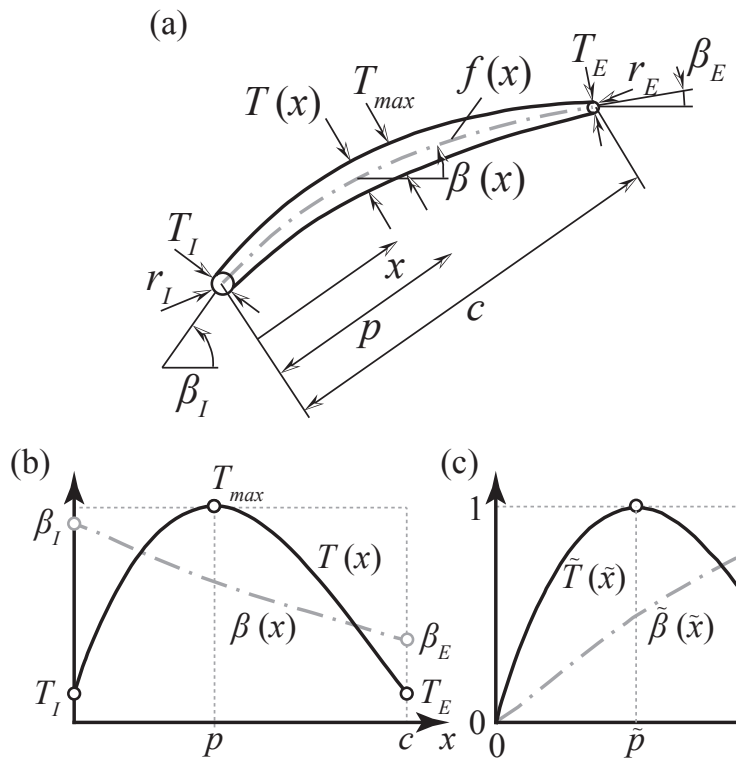

(c)

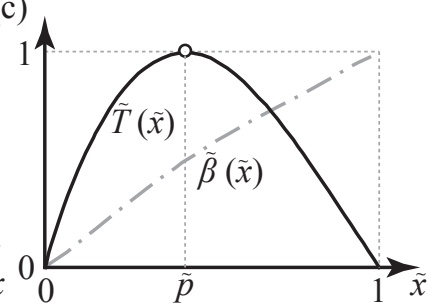

FIGURE 1: PARAMETERIZATION OF BLADE SECTION: (a) PROFILE SKELETON; (b) DIMENSIONAL AND (c) NON-DIMENSIONAL CAMBER-LINE ANGLE AND THICKNESS DISTRIBUTIONS.

\section{Blade Parameterization}

The $B S$ geometry is parameterized using Parablading and is defined by 21 individual $2 D$ profiles from hub to tip. Using a curve in the radial direction defined by axial and circumferential shifts, the design sections are stacked from the hub to the casing, resulting in the final $3 D$ aerofoil shape [27-29]. For each profile contour definition the classical section build-up is applied [30], which is originally based on the superposition of a camber-line $f(x)$ and a thickness distribution $T(x)$. However, instead of using the camber-line, the camber-line angle distribution $\beta(x)$ is preferred. In this work, the normalized distributions illustrated in Fig. 1 are parameterized by B-splines [31] using 7 control points $(C P)$ for the non-dimensional camber-line angle distribution and $9 \mathrm{CP}$ for the normalized thickness distribution.

From the 21 individual 2D profiles, 7 are selected as design sections and the rest are interpolated using splines. Thus, the potential design space dimension amounts to 196: 5 discrete design parameters $\left(r_{I}, r_{E}, \beta_{I}, \beta_{E}\right.$ and $\left.T_{\max }\right), 10$ coordinate parameters for the non-dimensional camber-line angle distribution, 11 coordinate parameters for the non-dimensional thickness distribution and 2 stacking parameters for the axial and circumferential shift (per section). However, the stacking shifts at hub and tip are kept constant, leading to a final (including constraints) design space of 192 parameters which were used by the adjoint-based optimization.

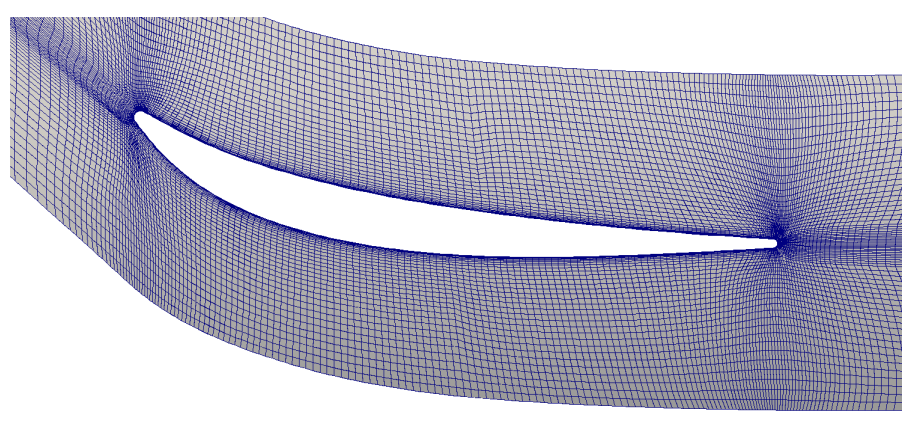

FIGURE 2: STATOR CFD MESH GENERATED IN PADRAM.

\section{Mesh Generation}

Figure 2 shows the block-structured 1.9 million node hexahedral mesh that was generated in PADRAM [32], the RollsRoyce in-house meshing tool, after conducting a mesh refinement study to achieve a mesh-independent prediction of the objective functions. The maximum first node dimensionless wall distance $y^{+}$value is below 4 , which is acceptable since an (inhouse coded) adaptive wall function technique is used in the $C F D$ simulation.

\section{Flow Simulation}

A steady RANS compressible flow solver with the SpalartAllmaras turbulence model is used to compute the flow. This is part of the Rolls-Royce in-house HYDRA [33] suite of codes, which has been extensively validated and applied to various industrial cases [32,34]. More details regarding the underlying theory and implemented algorithm can be found in [35, 36]. The nonlinear flow solver uses a node-based finite-volume discretization method and the pseudo-time-marching to steady state is accelerated by a block-Jacobi preconditioner and a geometric multigrid technique. Three primal/flow CFD simulations have been performed for the $B S$ blade (one per operating point). The mass flow constraint is satisfied by adjusting the outlet static pressure during the iterative solution until the prescribed mass flow is achieved. The experimentally observed corner flow separations occurring on both hub and tip are also captured by the simulation and have to be reduced in order to decrease the stators total pressure loss. The $D P$ total pressure loss coefficient distribution for the $B S$ at an axial cross-section close to the trailing edge is shown in Fig. 5. The lowest values appear at the areas of separated flow. After converging the flow $C F D$ simulations, the mass-averaged total pressure loss coefficient $\omega$ was computed for $D P, O D$ - and $O D+$ as shown in table 1 . This leads to a weighted $\omega_{w}$ of 0.0897 for the $B S$ blade. Finally, Fig. 6 shows the $D P$ axial whirl angle distribution at the outlet of the $C F D$ domain. It is noticed that the outlet whirl angle highly deviates from zero, which means that the $B S$ blade does not achieve the prescribed

Copyright () 2018 by Rolls-Royce Deutschland Ldt. \& Co KG 
flow turning. The mass-averaged exit flow angle deviation $\alpha_{E}$ has been calculated for $D P, O D$ - and $O D+$ conditions, shown in table 1. Thus, the weighted $\alpha_{E, w}$ for the $B S$ blade is $4.56^{\circ}$. The weighted $\omega_{w}$ and $\alpha_{E, w}$ are the two contradicting objectives which the stator should be optimized for.

TABLE 1: $C F D$ CALCULATED MASS-AVERAGED TOTAL PRESSURE LOSS COEFFICIENT $\omega$ AND EXIT FLOW ANGLE $\alpha_{E}$ FOR $B S$

\begin{tabular}{lll}
\hline Incidence & $\omega$ & $\alpha_{E}$ \\
\hline$O D-$ & 0.0829 & $3.87^{\circ}$ \\
$D P$ & 0.0843 & $4.45^{\circ}$ \\
$O D+$ & 0.1071 & $5.46^{\circ}$ \\
\hline
\end{tabular}

\section{Adjoint Simulation}

Given that two objective functions are considered for each operating point, six adjoint $C F D$ simulations need to be performed in total. This cannot be avoided, since each operating point has a different flow solution with which the adjoint problem is started. The converged adjoint solution was proven to be adequate to compute flow sensitivities leading to sufficiently accurate gradient information, after a finite-difference validation study was conducted similar to [24].

The adjoint solver provides the volume sensitivities (i.e. the change in objective function with respect to a volume mesh node perturbation). The surface sensitivities (i.e. the change in objective function with respect to a surface mesh node perturbation) are obtained using the inverse operation of a spring-based mesh deformation algorithm on the volume sensitivities. Finally, the adjoint surface sensitivity map is derived by projecting each surface node's sensitivity vector onto its corresponding boundary normal.

Figure 3 illustrates the weighted sensitivity map obtained by the weighted sum of the sensitivities from the different operating points and by subsequently weighting the two objectives (to be used in the following section). The numerical definition of the sensitivity is:

$$
\begin{aligned}
\frac{d F_{\text {aug }}}{d X_{n}} & =w \cdot\left[0.5 \cdot \frac{d \alpha_{D P}}{d X_{n}}+0.25 \cdot\left(\frac{d \alpha_{O D-}}{d X_{n}}+\frac{d \alpha_{O D+}}{d X_{n}}\right)\right] \\
& +0.5 \cdot \frac{d \omega_{D P}}{d X_{n}}+0.25 \cdot\left(\frac{d \omega_{O D-}}{d X_{n}}+\frac{d \omega_{O D+}}{d X_{n}}\right),
\end{aligned}
$$

where $d X_{n}$ stands for a discrete point perturbation in the surface normal direction and $w$ is chosen to be equal to 100 . Blue areas should be pulled outwards (and red pushed inwards) to achieve a decrease in the weighted augmented objective function. The black line denotes the zero sensitivity line.

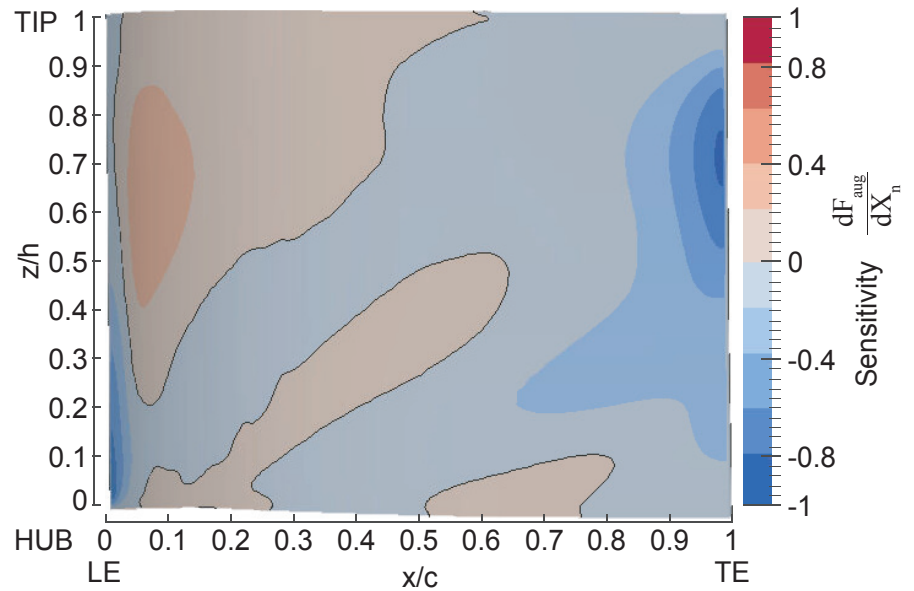

FIGURE 3: SUCTION SIDE OF $B S$ BLADE COLORED BY WEIGHTED SENSITIVITY MAP.

\section{ADJOINT-DRIVEN OPTIMIZATION}

The automated gradient-based optimization workflow which was developed in [25] is also used in this work. The $B S$ geometry is exported from Parablading and imported into PADRAM, where the $B S C F D$ mesh is generated. The HYDRA primal solver reads in this mesh and performs three flow simulations in parallel for the three different operating points. Subsequently, the HYDRA adjoint solver reads in the converged flow solutions and executes the six adjoint simulations in parallel, which result in six sensitivity maps $\left(d F / d X_{n}\right)$.

After the end of this process, the sensitivity maps as well as the $B S$ mesh are passed to Parablading again in order to calculate the final gradients $(d F / d \pi)$, i.e. the derivatives of the objective functions with respect to the design parameters. This first requires the computation of the geometric sensitivities $\left(d X_{n} / d \pi\right)$ for each design parameter, which is done inside Parablading using finite-differences. Following the chain rule, the gradients $d F / d \pi$ are computed by taking the inner product of each sensitivity map with each geometric sensitivity field. Although the computational cost of this approach scales with the number of design parameters, the total cost for the 192 parameters used in this case is negligible compared to the cost of the primal and adjoint solutions.

As a result, 6 gradient vectors of 192 components are obtained. The weighted $\omega_{w}$ gradient is derived using the same weighted sum for the gradient vectors of the different operating points that was used for the objective function values. Similarly, the weighted $\alpha_{E, w}$ gradient is computed.

Gradient-based optimizers deal with single-objective optimization problems. Thus, an augmented objective function is defined as the sum: $F_{\text {aug }}=\alpha_{E}+100 \cdot \omega$, which equally weights the two objectives $\left(\omega=0.0897, \alpha_{E}=4.56^{\circ}\right)$. This is selected as the

Copyright @ 2018 by Rolls-Royce Deutschland Ldt. \& Co KG 


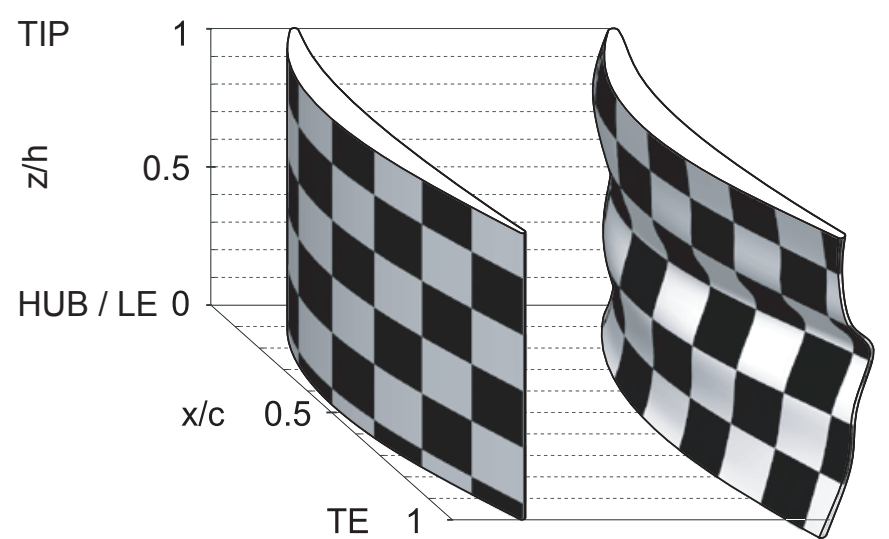

(a) BS

(b) OPS

FIGURE 4: COMPARISON OF STATOR SHAPES.

problem's cost function to be minimized starting from the baseline value $F_{\text {aug }}=13.52$. In order to be consistent, the gradient of $F_{\text {aug }}$ with respect to the design parameters is also given by the sum of $\omega_{w}$ and $\alpha_{E, w}$ gradients (Equation 2). Finally, this gradient information is passed to a steepest descent optimizer and it is used to update the design space and thus the $B S$ geometry. The whole process is wrapped in Python and is repeated until the optimization criteria are met.

The optimum is found in only seven design steps and $F_{\text {aug }}$ is decreased by $15.1 \%$. This results from a large decrease in $\alpha_{E, w}$ by $38.6 \%$ and a small reduction in $\omega_{w}$ by $3.2 \%$. The aforementioned two objectives are contradicting (the gradients point towards different directions at the optimized stator [OPS] blade), meaning that the optimization output varies for a different weighting of the objectives. Repeating the optimization for a number of possible weights may lead to a set of non-dominated solutions in objective space, forming the Pareto-front. The manufacturing constraints of the case have been met throughout the optimization using the same approach as in [25].

It is shown in Fig. 4 that the optimizer has come up with a leaned (bow-shaped) blade which is highly loaded at mid-span and less loaded at hub and tip, thus reducing the still existing corner flow separations. This is also illustrated in Fig. 5, where the $D P$ total pressure loss coefficient distribution at an axial crosssection just behind the trailing edge is plotted for both $B S$ (left) and $O P S$ (right) blades. The weighted objective functions for the OPS blade are $\omega_{w}=0.0868$ and $\alpha_{E, w}=2.8^{\circ}$.

Figure 6 shows the $D P$ axial whirl angle distribution at the outlet of the $C F D$ domain for both $B S$ (left) and $O P S$ (right) blades. The $O P S$ blade achieves a flow angle deviation from the axial direction closer to zero. In order to obtain a more uniform angle profile, the standard deviation value would also need to be included in the objective function. The final blade geome-

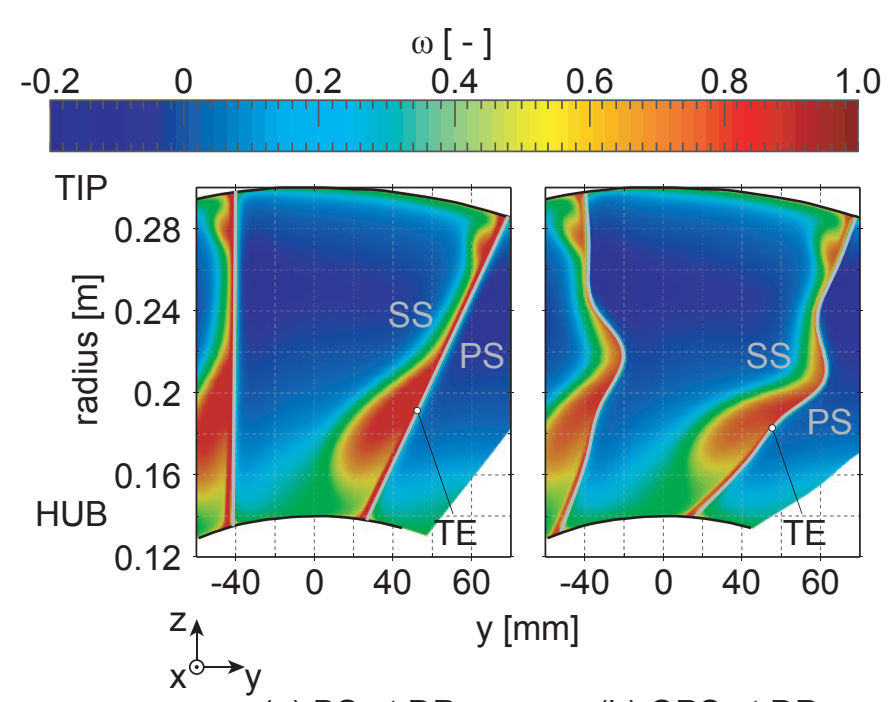

(a) BS at DP

(b) OPS at DP

FIGURE 5: CFD RESULTS OF THE TOTAL PRESSURE LOSS COEFFICIENT DISTRIBUTION AT THE TRAILING EDGE.

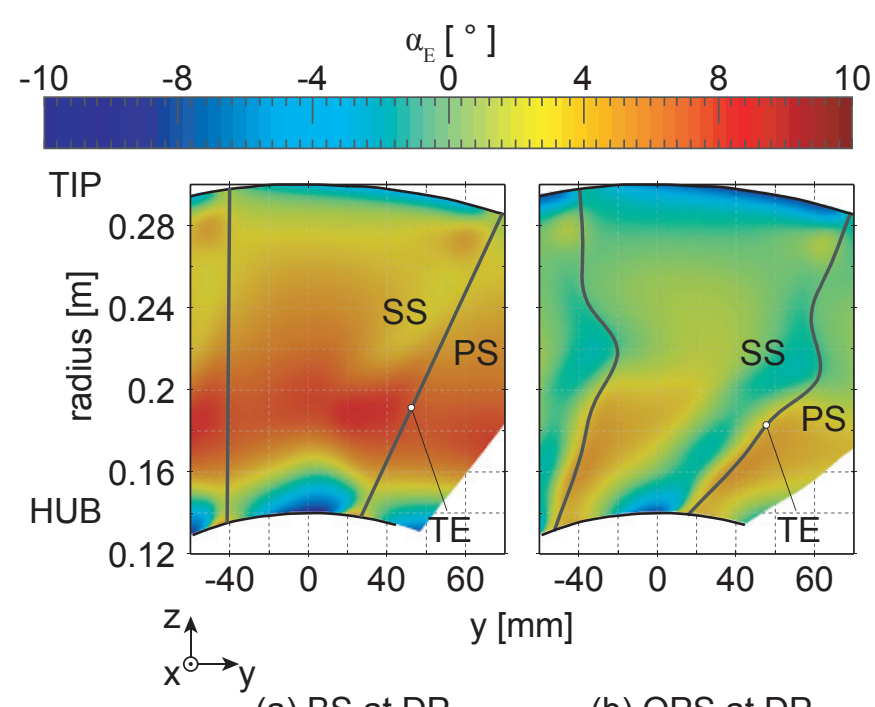

(a) BS at DP

(b) OPS at DP

FIGURE 6: $C F D$ RESULTS OF THE EXIT WHIRL ANGLE DISTRIBUTION AT THE WAKE PLANE $\left(x=0.6 \cdot c_{S}\right)$.

try which was optimized using the Spalart-Allmaras turbulence model was also validated with the $k-\omega$ SST model, i.e. obtained similar improvements in both objectives for the two setups.

Copyright @ $\odot 2018$ by Rolls-Royce Deutschland Ldt. \& Co KG 


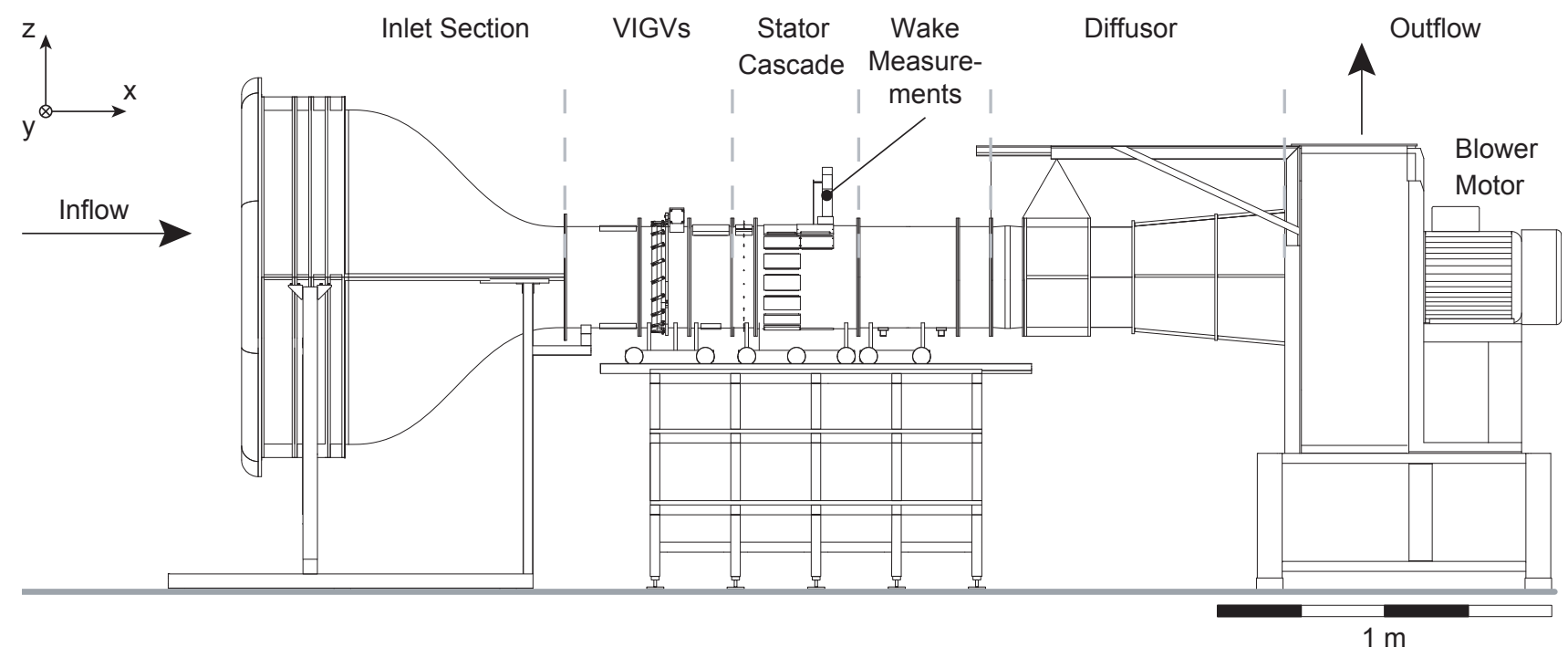

FIGURE 7: FULL ANNULAR TEST RIG AT TU BERLIN

\section{EXPERIMENTAL SETUP AND PROCEDURE}

All experimental investigations were carried out using a low speed, open circuit wind tunnel at the Chair for Aero Engines of the TU Berlin. The wind tunnel is used for experimental investigations as part of the collaborative research center $(C R C) 1029$ [37]. Its main objective is the investigation of the influence of a pressure-gaining combustion on compressor components. Current projects focus on the stator sections of a compressor. The annular design was chosen to create realistic three dimensional flow characteristics and thus to enable investigation of the effects of a $P D E$ at a high spatial resolution.

Fig.7 shows a schematic depiction of the wind tunnel. A round inlet nozzle with a contraction of $11: 1$ is used to create smooth inflow conditions and low turbulence intensity by means of screens and honeycombs as flow straighteners. When reaching the main casing dimensions of $0.6 \mathrm{~m}$ a variable inlet guide vane $(V I G V)$ produces the swirl needed for the stator inlet conditions. Using 19 turbine shaped blades, a turning of $42^{\circ}$ is achieved at midspan. Moreover, these VIGVs provide the option of changing the incidence to the stator by $\pm 5^{\circ}$.

The annular measurement section consists of a highly loaded compressor stator cascade. The $B S$ profile is a controlled diffusion airfoil. The blades are designed to produce an axial outflow at design point $(D P)$ with a chord-based Reynolds number of $R e=6 \times 10^{5}$ without any gap at hub or tip and with a total mass flow of $\dot{m}=9.5 \mathrm{~kg} / \mathrm{s}$.

The axial distance between the VIGV and the stator inlet is about three chord lengths to ensure a good mixing of the blade wakes and producing an inlet turbulence of $T u_{I}=5 \%$. The degree of turbulence for the flow was measured by means of hot wire anemometry at the leading edge of the stator profiles. Fig. 8 de-

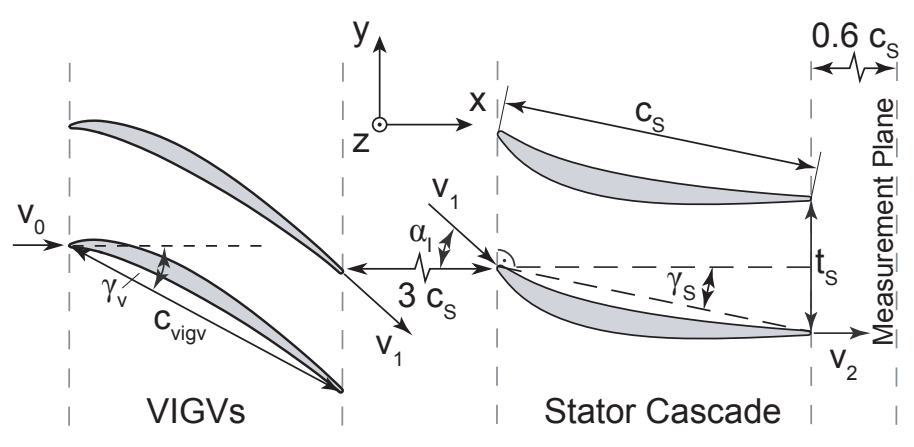

FIGURE 8: $V I G V$ AND STATOR GEOMETRY

picts the geometric data and is complemented by Table 2. For the oil-flow visualization a mixture of liquid paraffin oil and dye powder was used. The flow at the inlet and wake of one stator passage was measured with a miniature five-hole probe using differential pressure sensors (First Sensor; HDO-Type) with a calibrated pressure range of -50 to 50 mbar. The inlet plane is located $1.4 \cdot c_{s}$ upstream of the $L E$ of the stator. The wake plane measurements were taken at $0.6 \cdot c_{s}$ downstream of the stators $T E$ (see Fig. 8). In order to get a detailed pressure distribution and velocity profile, the five-hole probe was traversed to 300 points in a circumferential based polar grid, measuring mean values at each location. The grid has 20 equidistant radial lines. On each radial line grid points are distributed equidistantly along the circumference. The radial line at hub side holds 10 points while the radial line at tip side holds 20 points.

Copyright @ 2018 by Rolls-Royce Deutschland Ldt. \& Co KG 
TABLE 2: GEOMETRIC DATA OF THE ANNULAR TEST RIG

\begin{tabular}{lll}
\hline Name & Parameter & Value \\
\hline$V I G V$ blade count & $n_{V I G V}$ & 19 \\
Stator blade count & $n_{S}$ & 15 \\
Stator chord length & $c_{S}$ & $187.5 \mathrm{~mm}$ \\
Stator height & $h_{S}$ & $150 \mathrm{~mm}$ \\
Stator turning & $\Delta \alpha$ & $42^{\circ}$ \\
Hub to tip ratio & $r_{h u b} / r_{t i p}$ & 0.5 \\
Pitch to chord ratio midspan & $t / c$ & 0.5 \\
De Haller & $v_{2} / v_{1}$ & 0.5 \\
\hline
\end{tabular}

\section{Inflow Conditions}

The inflow conditions for the present experiments have been numerically calculated using the ANSYS CFX framework in order to mimic a generic rotor model. Said rotor has been designed using the radial equilibrium while meeting the geometric constraints of the wind tunnel and not exceeding the available propulsive power. The optimization of the $B S$ profiles with regard to a generic rotor wake makes the $O P S$ stator usable in a future wind tunnel configuration. On the basis of the simulated rotor wake flow, a VIGV profile has been designed using the work of Banjac et. al. [38]. For the experiment conducted herein, the $V I G V \mathrm{~s}$ are designed as three-dimensional blades by means of RANS simulations to ensure an inlet flow angle profile $\alpha_{I}=f(z)$ along the stator blade height. While replicating the mean rotor inflow conditions of the above mentioned generic rotor, using this $V I G V$ design, the main focus was set onto the area between $10 \%$ and $90 \%$ of the passage height. Because of the static hub and the nonexisting clearance at the tip, the increasing deflection - especially in the hub area - could not be met. Additionally, the inflow experiments were performed at $O D$ - and $O D+$. These incidence shifts are of interest for future studies as the shifting inflow conditions under transient boundary conditions of a pulsating combustion require an aerodynamically robust profile.

Fig. 9 depicts the circumferentially-averaged inflow angle $\alpha_{I}$ on the stator versus the stator passage height. The measured angle displacements for the $O D$ - and $O D+$ incidence variations are shown. These inflow conditions have been used in the experi-

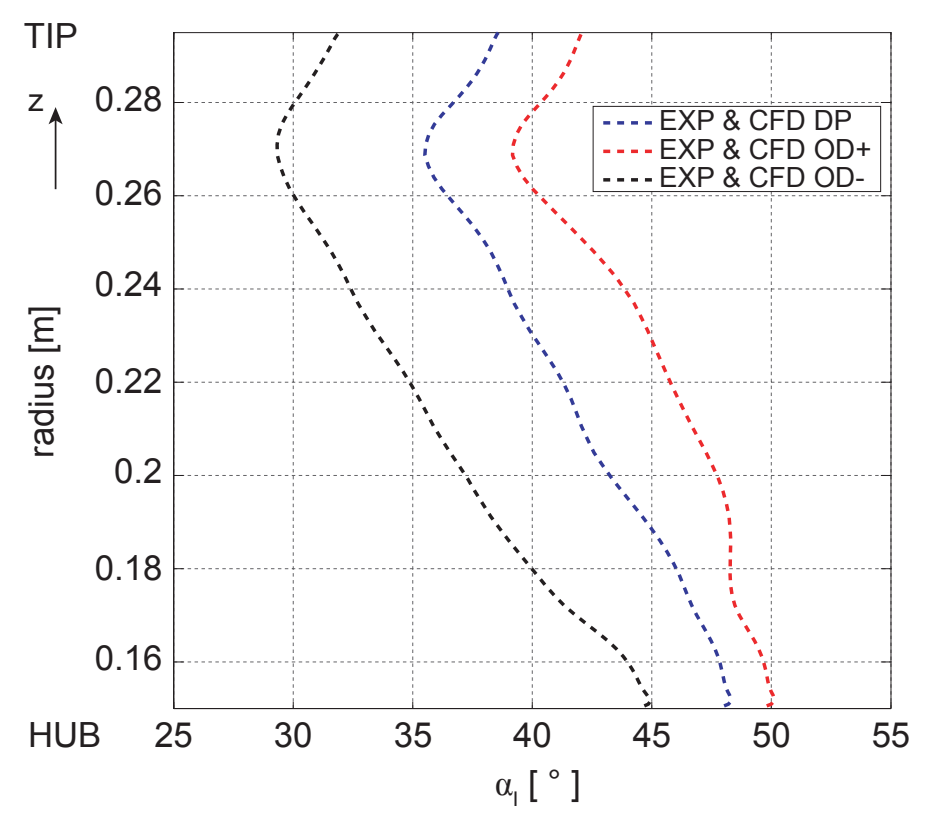

FIGURE 9: CASCADE INFLOW CONDITIONS FOR THE INVESTIGATED STATOR SETS

mental and the numerical investigations.

\section{RESULTS AND DISCUSSION}

This section discusses oil-flow visualizations displaying flow quality magnitudes, vector and particle traces on the stator sets under investigation. These visualizations of the flow field provide accuracy checks and additional understanding of the underlying flow physics. The comparative visualizations of the oilflow experiments were carried out under the same inflow conditions using the same incidence variations of $O D-, D P$ and $O D+$. Subsequently, five-hole probe pressure measurements in the wake of the stator blades are discussed and evaluated to visualize the flow characteristics for one passage. These measurements are then compared in detail against the obtained $C F D$ results.

\section{Oil flow Visualizations}

The results presented in the Figures 10 through 12 provide a comparative depiction of oil-flow visualizations and $C F D$ simulation results. The streaklines indicate the near wall flow direction on the SS. The oil-flow visualizations of the incidence variations of the $B S$ set show large areas of 3D separations (Figures 11a, 10a and 12a) clearly indicated by reverse flow (negative axial velocities) [7]. Separation lines, marked by the convergence of neighboring streaklines, can be identified on the SS of the BS and OPS. Unsteady movement of two identified focus points (marked as red dots) form an area of recirculating flow 


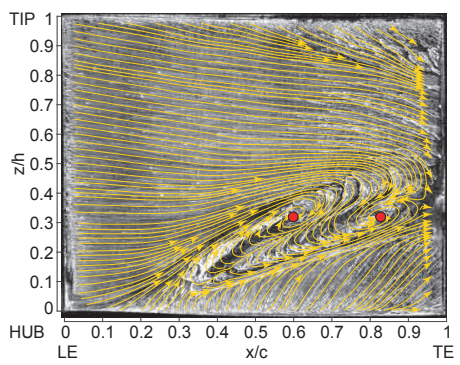

(a) Oil-Flow Visualization $(B S)$

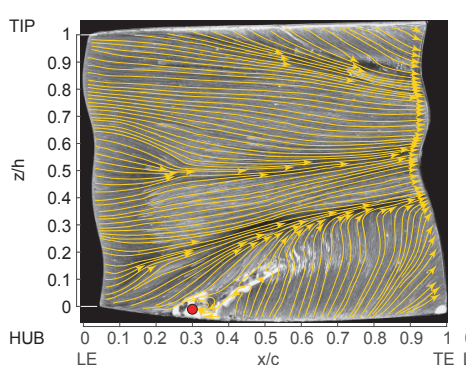

(c) Oil-Flow Visualization (OPS)

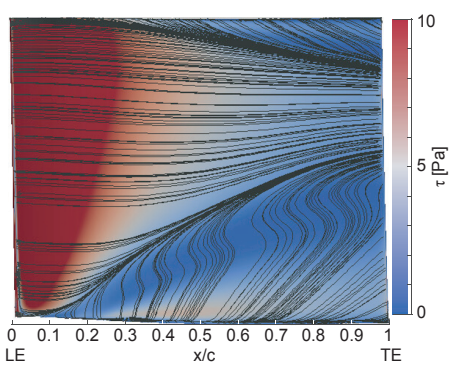

(b) CFD-Surface Streamlines $(B S)$

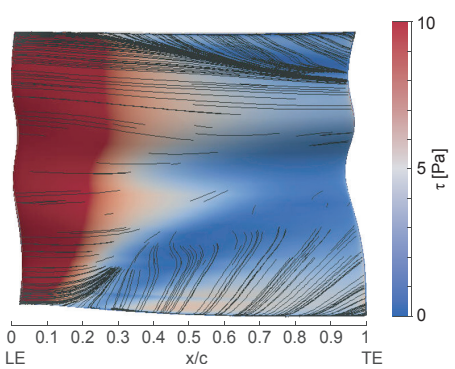

(d) CFD Surface Streamlines (OPS)
FIGURE 10: RESULTS ON THE SS FOR BOTH STATORS AT DP

structure which appears in a more or less distorted form on all three investigated incident cases of the $B S$ (SS). As the $C F D$ calculations are steady, any unsteady effects in the flow field are suppressed. Hence, unsteady secondary flow structures are not visible in the $C F D$ results presented in the Figures 10,11 and 12. Furthermore, a clear definition of the separation line for the $C F D$ results of the $O P S$ is hard due to missing streaklines in the midsection. However, since separation from the $S S$ occurs where the shear stress vanishes, the additional depiction of the wall shear stress distribution $\tau_{w}$ provides further insight on separation line formation. Nonetheless, the areas of undisturbed main flow are distinctively present in the oil-flow patterns and the $C F D$ results for both stator sets. Characteristic areas can be relocated in both results - oil-flow patterns and $C F D$-, the flow topologies in these areas are very distinct though.

BS DP: The focus points, most visible at the $D P$ case of the $B S$ (see Fig. 10a), move in a mainly diagonal direction due to highly transient flow phenomena. Caused by their diagonal occurrence, that is to say, the focal points movement alongside the separation line, these secondary flow structures acquire more oil-paint than surrounding flow structures resulting in an area of glazed oil-paint. Figure 10a shows a stable corner vortex developing at $20 \%$ chord length from the $L E$ and extending until the $T E$. Between this corner vortex and the main flow, a recirculation area is formed where the above mentioned focus points reside. The corner separation is fed by the main flow. The main flow is distorted towards the hub side at the trailing edge which is caused

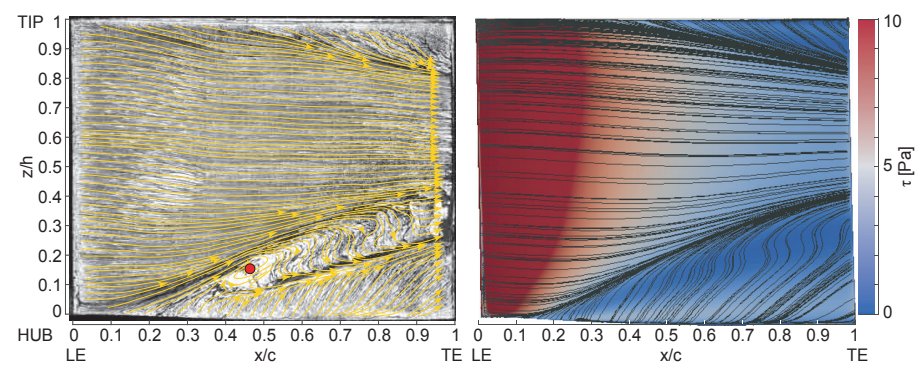

(a) Oil-Flow Visualization $(B S)$

(b) CFD Surface Streamlines (BS)

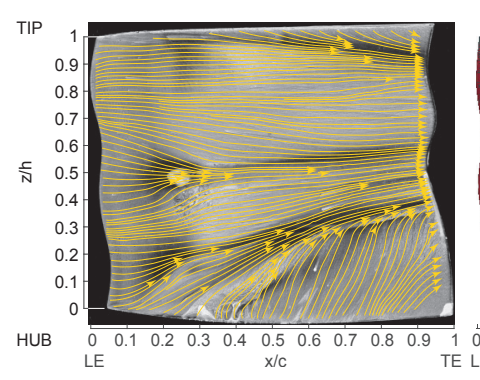

(c) Oil-Flow Visualization (OPS)

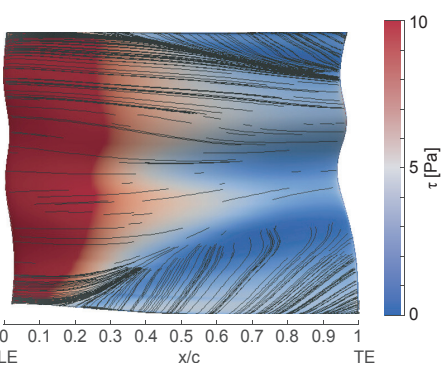

(d) CFD Surface Streamlines (OPS) by the recirculation area, an effect which is not visible in the corresponding $C F D$ results.

BS OD-: In Fig. 11a only one focal point is to be identified in the recirculation area at $46 \%$ chord length from the $L E$ and $15 \%$ chordlenght from the hub wall. This focal point has moved upstream and in hub direction when compared to the $D P$ case of the $B S$. Due to the compressed and distorted nature of the focal points flow field, the second focal point moved to a virtual point beyond the trailing edge. Secondary flow, caused by the corner vortex system, extends less in radial direction compared to the $D P$ case. The main flow is less influenced by secondary flow phenomena, which is in fair agreement with the $C F D$ results shown in Figure 11b.

BS OD+: Figure 12a shows the focal points at $31 \%$ and $49 \%$ chord length from the $L E$ and $24 \%$ as well as $20 \%$ chordlenght from the hub wall respectively. Their flow structure is clearly expanded in radial direction forming areas of large recirculation. The corner vortex separation lines starting point has significantly moved upstream compared to Figures 10a and $11 \mathrm{a}$, caused by an increased axial pressure gradient acting from the $T E$ direction. The corner vortex developing at $10 \%$ chord length seems to break down (burst) at 55\% chord length from the $L E$, as the streaklines in Fig. 12a indicate that fluid from the boundary layer is no longer transported into the vortex system, but detaching and migrating into the recirculation area. Thus, starting from $60 \%$ chord length from the $L E$ the corner flow is no longer part of the corner vortex indicating an energy loss of 


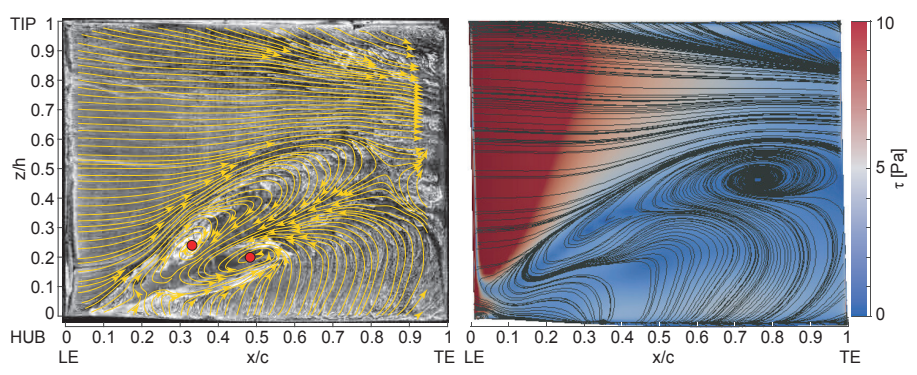

(a) Oil-Flow Visualization $(B S)$

(b) CFD Surface Streamlines $(B S)$

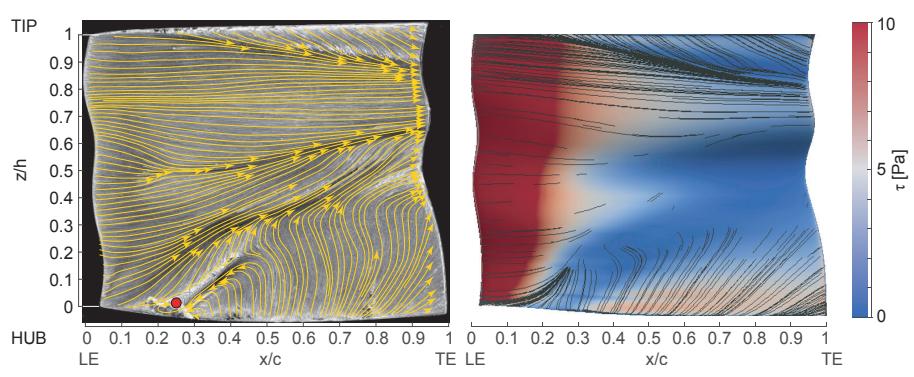

(c) Oil-Flow Visualization (OPS)

(d) CFD Surface Streamlines (OPS)

FIGURE 12: RESULTS ON THE SS FOR BOTH STATORS AT OD+

the corner vortex system.

For all three results of the oil-flow visualization of the $O P S$ profile it can be seen in Figures 11c, 10c and 12c that a significant reduction of secondary flow structures on the $S S$ is evident. The radial extension of secondary flow structures has been reduced and recirculation in axial direction is suppressed significantly. The resulting streaklines are more guided in axial direction and a much larger percentage of the main flow field on the $S S$ of the $O P S$ is contributing to a flow in $T E$ direction. The point of origin for a starting corner vortex separation can be placed to $15 \%$ chord length from the $L E$ and is fixed in its position in all incidences for the $O P S$ (Figure 11c, 10c and 12c), which is consistent with the CFD results.

\section{Wake Measurements}

Figure 13 shows the measured total pressure loss coefficient $\omega$ for all three incidences of the $B S$ at a distance of $0.6 \cdot c_{s}$ downstream the $T E$. The topology of the measurement plane is approximately a passage width between two stator blades. Starting as a corner vortex and gaining spatial expansion over the $S S$ length, the dominating corner separation can be clearly identified (see Fig. 11a, 10a and 12a). The 3D separation region on the $S S$ is increasing with $i$ causing a growing area of total pressure loss in the wake. While acting as a blockage for the cross-section flow of the passage, the expanded secondary flow structures are causing an increased exit whirl in the tip region. Effects of the profile's wake are clearly visible in all incidence plots of Figure 13 but are diminished with raising incidence. The tip sided corner vortex is smaller than the hub sided one as the lateral pressure gradient is smaller due to a larger blade pitch at the tip. Furthermore, the passage flow deflection in the casings direction is causing a constriction of the corner vortex.

The wake measurements of the total pressure loss coefficient $\omega$ for the $O P S$ set is depicted in Fig.15. This Figure shows clearly defined local differences in the secondary flow structures when compared to the $B S$ measurements. A necessary circumferential offset of the two different profiles in counter-clockwise direction around the $\mathrm{x}$-axis - while keeping the measurement grid constant - may account for these differences to a certain extent. As expected and according to the $O P S$ profile, the measured $T E$ wake shows a curved tendency towards the tip direction. The corner separation in the wake flow of the $O P S$ shows similar effects as the $B S$ regarding its spatial expansion and increasing total pressure loss with increasing incidence. All Figures ( $B S$ and $O P S$ ) show an increase of $\omega$ in the vicinity of the hub-based corner separation core. However, the measured peak loss values of the total pressure loss coefficient $\omega$ for the OPS in that region are significantly lower than for the $B S$ case. Outside of the detachment zone the measured $O P S$ profile shows a slightly increased $\omega$ over a large region of the passage height. This is especially evident in the $O D+$ case of the $O P S$. The radial position of the measured peak loss values within the corner separations core are nearly the same and no deviation can be observed. The loss coefficient is below zero in the boundary region of the measurement plane which is unphysical but can be explained with the measurement methods used in the experiments. The five-hole probe causes a nozzle flow with the probe head in the vicinity of the side walls causing the data to migrate outside the calibration range and thus resulting in a total pressure which is too high. Furthermore it may be noted, that a minor circumferential shift between the inflow measurement plane and the outflow measurement plane had to be introduced due to mechanical restrictions of the traversing system. This results in a redistribution of flow impulse, introducing an error in the computation of the pressure loss coefficient.

\section{Comparison between CFD Results and Measurements}

A comparative depiction of the pressure loss coefficient obtained by the $C F D$ calculation is shown in Figure 14 and 16 . While the measurement plane is highlighted in these Figures, a larger plane is provided in transparency for convenient comparison of the flow phenomena. The $C F D$ results are in fair agreement with the measured results presented in Figures 13 and 15. The predicted location of corner separation induced pressure loss can be confirmed by these results. Although the overall appear-

Copyright @ (c) 2018 by Rolls-Royce Deutschland Ldt. \& Co KG 


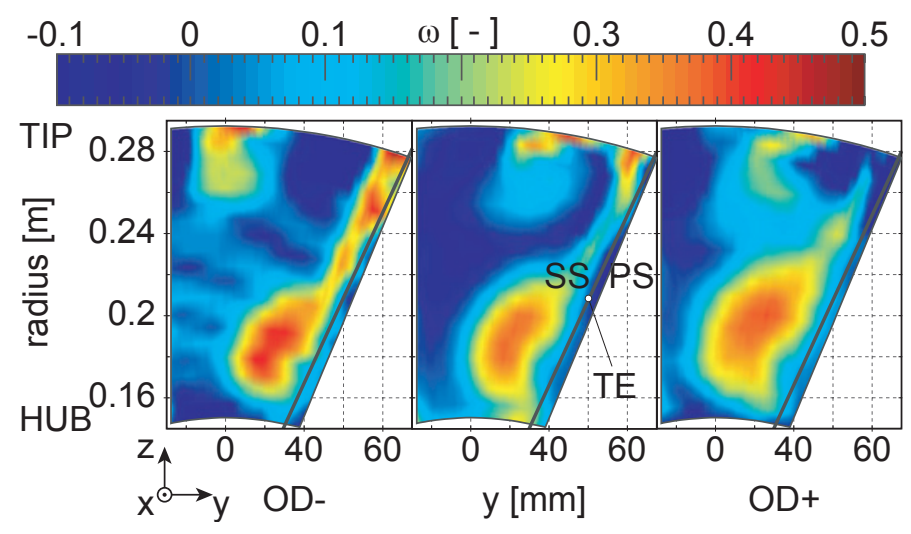

FIGURE 13: EXPERIMENTAL MEASURMENTS OF THE TOTAL PRESSURE LOSS COEFFICIENT $\omega$ IN THE WAKE OF THE $B S$

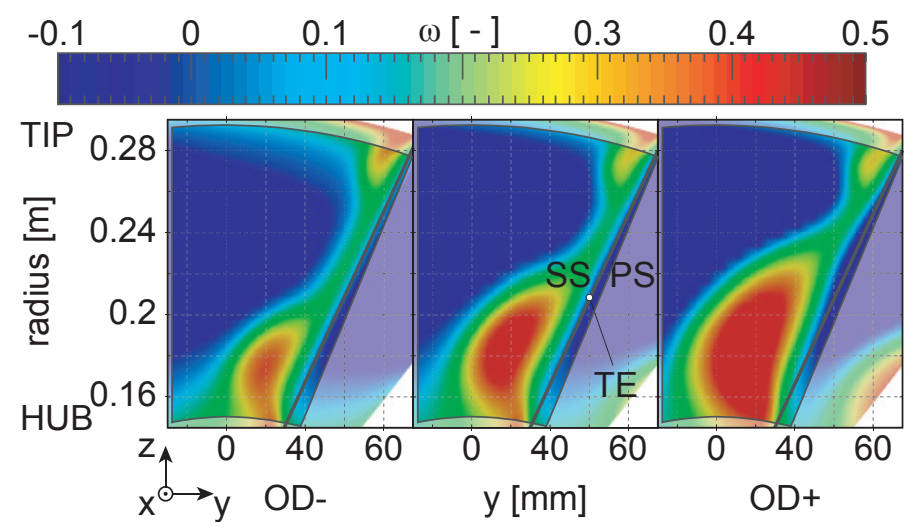

FIGURE 14: $C F D$ RESULTS OF THE TOTAL PRESSURE LOSS COEFFICIENT $\omega$ IN THE WAKE OF THE $B S$

ance of the loss pattern is similar for the $C F D$ predictions and measurements, the measured trailing edge wake of the $B S$ and $O P S$ is not as smooth as predicted. The measured hub sided corner stall regions are more diffused in the radial direction than they are in the $C F D$ result. Furthermore, the measured peak loss values are significantly lower than the numerical results in both the $B S$ and $O P S$ case. The regions of pressure loss in the OPS wake are decreased in the circumferential as well as in the radial direction when compared with the $B S$ wake. These results are in accordance between the $C F D$ predictions and the measured data. A comparison between the mass-averaged total pressure loss coefficient $\omega$ and the mass-averaged exit whirl angle $\alpha_{E}$ obtained from the $C F D$ calculation and the measured data for all incidences is shown in Figure 17. The exit whirl angle shows a significant improvement from the $B S$ to the $O P S$, while the predicted and the measured data are in good agreement. The axial offset of the exit whirl angle was reduced by approximately $3^{\circ}$ for all incidences. The mass-averaged pressure loss coefficient

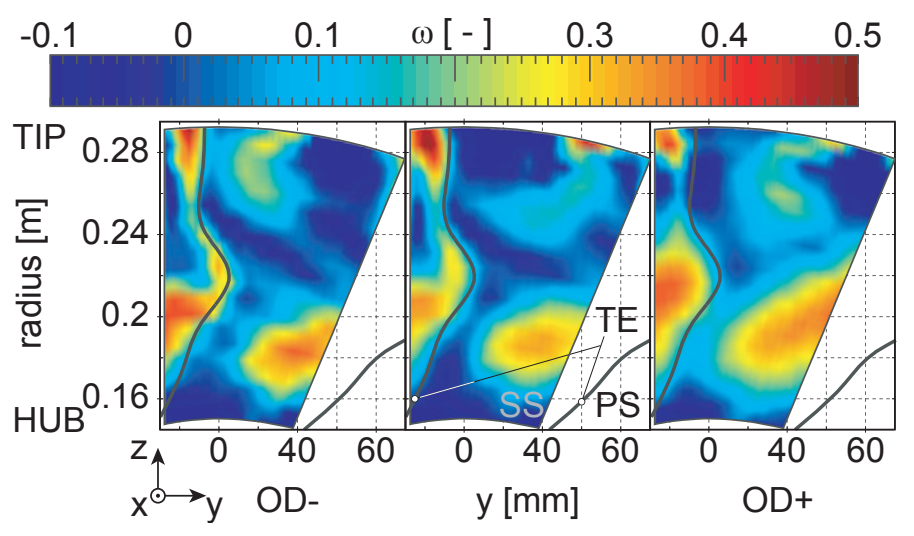

FIGURE 15: EXPERIMENTAL MEASURMENTS OF THE TOTAL PRESSURE LOSS COEFFICIENT $\omega$ IN THE WAKE OF THE $O P S$

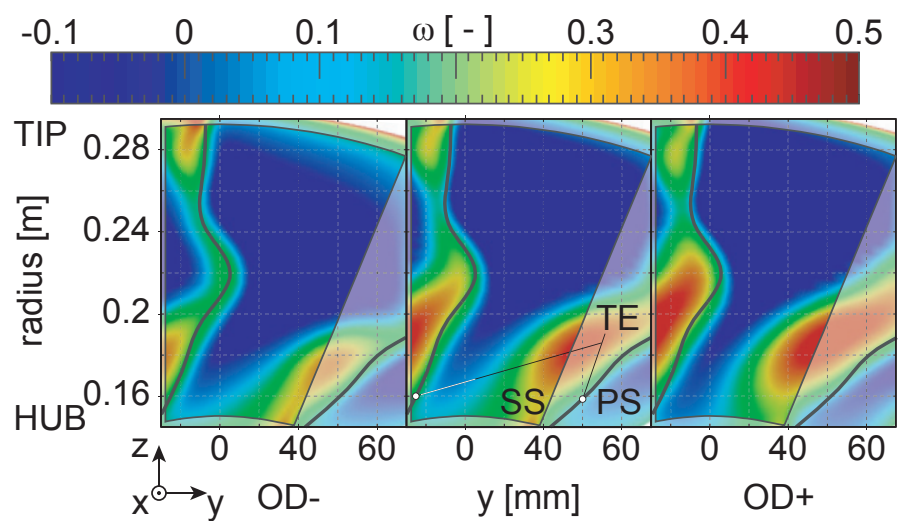

FIGURE 16: $C F D$ RESULTS OF THE TOTAL PRESSURE LOSS COEFFICIENT $\omega$ IN THE WAKE OF THE OPS

shows fair agreement between the predicted and the measured results and a reduction in pressure loss for the $O P S$ is evident for the $O D$ - and the $D P$ case. However, a rise in averaged pressure loss is measured for the $O D+$ case, contrary to the CFD prediction. The $C F D O D+$ simulation for the $B S$ should not be trusted due to the high unsteadiness that exists and is being suppressed by the steady flow assumption.

\section{CONCLUDING REMARKS}

An optimization of the TU Berlin TurboLab Stator test case with subsequent experimental measurements and $C F D$ simulations was described. The presented results include stator blade $S S$ oil-flow visualizations, total pressure loss and flow angle distributions at the cascade exit plane for both the $B S$ and the $O P S$ case. These were compared against results from corresponding $C F D$ simulations. The following conclusions can be drawn from this study:

Copyright @ $\odot 2018$ by Rolls-Royce Deutschland Ldt. \& Co KG 


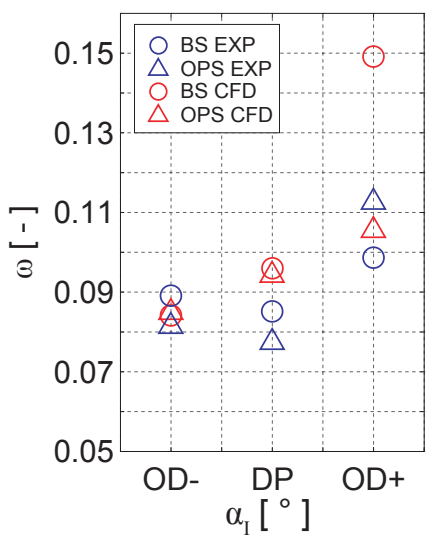

(a) PRESSURE LOSS COEFFICIENT

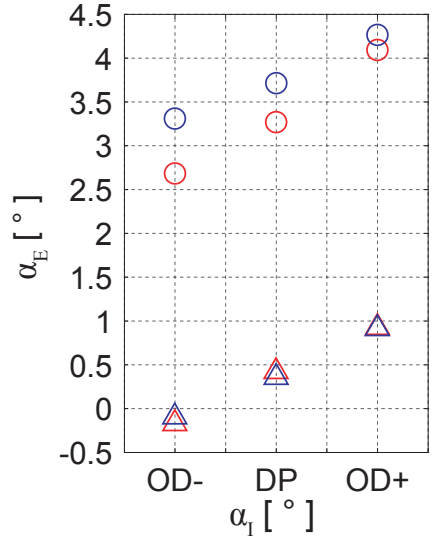

(b) OUTFLOW ANGLE
FIGURE 17: COMPARISON OF MASS-AVERAGED EXPERIMENTAL DATA AGAINST MASS-AVERAGED CFD RESULTS IN THE WAKE OF THE $B S$ AND THE $O P S$

1. An optimized airfoil was designed using an adjoint driven optimization which yielded a $O P S$ geometry having a thicker tip and hub section. Thus, manufacturing constraints such as fixture holes could be satisfied. Moreover, the formation of fillets at the hub does advantageously reduce corner separation. The OPS mid-span section is thinner compared to the $B S$ design, causing lower skin friction losses. The negative dihedral of the $O P S$ at mid-span results in a bowed stator decreasing the blade losses. A higher radius of curvature at the $L E$ of the $O P S$ allows for a general robustness against incidence variations in the $O D$ - and $O D+$ points. In general, the optimized airfoil of the OPS provides a more loaded blade while at the same time being less front-loaded compared to the $B S$.

2. The presence of corner separation for both stator sets was detected numerically and experimentally. It is known that the thickness of the separated region measured normal to the solid surface causes a significant blockage of the cross sectional flow area. It was shown that the OPS reduces these separated regions effectively.

3. It was found that, as the incidence onto the $B S$ and the $O P S$ blades was increased, the size of the separated region normal to the surface grew. However, the growth magnitude was much smaller for the OPS. These findings were in qualitative agreement of the measured and numerically obtained data.

4. In general, the numerical predictions of the optimization were found to be in good agreement with the experimental measurements, documenting the application of a gradientbased optimization workflow to a practical engineering problem. Based on the presented numerical and experimen- tal studies, it was shown that 3D blade optimization is an effective means of passive flow control. Separations in the stator passages were advantageously minimized and a stator airfoil design was achieved, providing a general robustness to changes in the inlet flow angle. These results show the general applicability of a passive flow control concept for stator blades in unsteady turbomachinery such as a $P D E$.

\section{ACKNOWLEDGMENT}

The authors gratefully acknowledge support by the Deutsche Forschungsgemeinschaft (DFG) as part of collaborative research centre CRC 1029 "Substantial efficiency increase in gas turbines through direct use of coupled unsteady combustion and flow dynamics". This work was conducted within the IODA project [22], funded by the European Union HORIZON 2020 Framework Programme for Research and Innovation under Grant Agreement No. 642959. The authors would like to thank RollsRoyce Deutschland for the permission to publish the work.

\section{NOMENCLATURE}

c chord length

F objective Function

h height

i incidence

$\dot{m} \quad$ mass flow

$\mathrm{p}$ pressure

$\mathrm{r}$ radius

Re Reynolds number

$\mathrm{T}$ Thickness

t pitch

$\mathrm{Tu} \quad$ turbulence intensity

$\mathrm{v} \quad$ velocity

$\mathrm{x}, \mathrm{y}, \mathrm{z} \quad$ coordinates / directions

$\mathrm{X}$ mesh node coordinate vector

\section{Greek}

$\alpha$

$\beta \quad$ blade (metal) angle

$\gamma \quad$ stagger angle

$\mu \quad$ dynamic viscosity

$\pi \quad$ design parameter

$\rho$ density

$\tau \quad$ shear stress

$\omega \quad$ total pressure loss coefficient

\section{Subscripts}

aug augmented

E Exit 


$\begin{array}{ll}\text { I } & \text { Inflow } \\ \max & \text { maximum } \\ \mathrm{n} & \text { normal } \\ \mathrm{S} & \text { stator } \\ \mathrm{t} & \text { total value } \\ \mathrm{w} & \text { weighted }\end{array}$

\section{Abbreviations}

$\begin{array}{ll}\text { 2D } & \text { two-dimensional } \\ \text { 3D } & \text { three-dimensional } \\ \text { BS } & \text { Baseline Stator } \\ \text { CFD } & \text { Computational Fluid Dynamics } \\ \text { CP } & \text { Control Points } \\ \text { CRC } & \text { Collaborative Research Center } \\ \text { DP } & \text { Design Point } \\ \text { EXP } & \text { Experimental } \\ \text { LE } & \text { Leading Edge } \\ \text { OD+ } & \text { Off-Design operating point with }+5^{\circ} \text { incidence } \\ \text { OD- } & \text { Off-Design operating point with }-5^{\circ} \text { incidence } \\ \text { OPS } & \text { Optimized Stator } \\ \text { PS } & \text { Pressure Side } \\ \text { PDE } & \text { Pulse Detonation Engine } \\ \text { RANS } & \text { Reynolds-Averaged Navier-Stokes } \\ \text { SS } & \text { Suction Side } \\ \text { TE } & \text { Trailing Edge } \\ \text { VIGV } & \text { Variable Inlet Guide Vane }\end{array}$

\section{REFERENCES}

[1] Shepherd, J., and Wintenberger, E., 2004. "Thermodynamic analysis of combustion processes for propulsion systems". In 42nd AIAA Aerospace Sciences Meeting and Exhibit, Aerospace Sciences Meetings. American Institute of Aeronautics and Astronautics.

[2] Shepherd, J., and Wintenberger, E., 2006. "Thermodynamic cycle analysis for propagating detonations". Journal of Propulsion and Power, 3(22), pp. 694-697.

[3] Staats, M., and Nitsche, W., 2016. "Active control of the corner separation on a highly loaded compressor cascade with periodic nonsteady boundary conditions by means of fluidic actuators". Journal of Turbomachinery, 138(3), p. 031004.

[4] Beselt, C., Eck, M., and Peitsch, D., 2014. "Three-dimensional flow field in highly loaded compressor cascade". Journal of Turbomachinery, 136(10), pp. 101007-1 - 101007-10.

[5] Cumpsty, N. A., 2004. Compressor aerodynamics, reprint ed. 2004 w/new preface, introduction and updated bibliography ed. Krieger, Malabar, Fla.

[6] Ma, W., Ottavy, X., Lu, L., Lebeouf, F., and Gao, F., 2011. "Experimental study of corner stall in a linear compressor cascade". Chinese Journal of Aeronautics, 24(3), pp. 235-242.
[7] Gbadebo, S. A., Cumpsty, N. A., and Hynes, T. P., 2005. "Threedimensional separations in axial compressors". Journal of Turbomachinery, 127(2), p. 331.

[8] Brück, C., Tiedemann, C., and Peitsch, D., 2016. "Experimental investigations on highly loaded compressor airfoils with active flow control under non-steady flow conditions in a 3d-annular lowspeed cascade". In Proceedings of the ASME Turbo Expo: Turbine Technical Conference and Exposition - 2016, The American Society of Mechanical Engineers, p. V02AT37A027.

[9] Lord, W., MacMartin, D., and Tillman, G., 2000. "Flow control opportunities in gas turbine engines". In Fluids 2000 Conference and Exhibit, American Institute of Aeronautics and Astronautics.

[10] Hecklau, M., Gmelin, C., Nitsche, W., Thiele, F., Huppertz, A., and Swoboda, M., 2011. "Experimental and numerical results of active flow control on a highly loaded stator cascade". Proceedings of the Institution of Mechanical Engineers, Part A: Journal of Power and Energy, 225(7), pp. 907-918.

[11] Zander, V., Hecklau, M., Nitsche, W., Huppertz, A., and Swoboda, M., 2011. "Active flow control by means of synthetic jets on a highly loaded compressor cascade". Proceedings of the Institution of Mechanical Engineers, Part A: Journal of Power and Energy, 225(7), pp. 897-908.

[12] Wang, X., Zhao, X., Li, Y., Wu, Y., and Zhao, Q., 2014. "Effects of plasma aerodynamic actuation on corner separation in a highly loaded compressor cascade". Plasma Science and Technology, 16(3), pp. 244-250.

[13] Peacock, R. E., 1965. Boundary-Layer Suction to Elimi- nate Corner Separation in Cascades of Aerofoils. Ministry of Defense,, Reports and Memoranda No. 3663.

[14] Gbadebo, S. A., Cumpsty, N. A., and Hynes, T. P., 2008. "Control of three-dimensional separations in axial compressors by tailored boundary layer suction". Journal of Turbomachinery, 130(1), p. 011004.

[15] Hergt, A., Meyer, R., Müller, M. W., and Engel, K., 2008. "Loss reduction in compressor cascades by means of passive flow control". In Proceedings of the ASME Turbo Expo 2008, ASME, pp. 269-280.

[16] Giannakoglou, K. C., Karakasis, M. K., and Kampolis, I. C., 2006. "Evolutionary algorithms with surrogate modeling for computationally expensive optimization problems". In Proceedings of the ERCOFTAC Design Optimisation: Methods \& Application Conference.

[17] Yamazaki, W., and Mavriplis, D. J., 2013. "Derivative-enhanced variable fidelity surrogate modeling for aerodynamic functions". AIAA Journal, 51(1), pp. 126-137.

[18] Giles, M. B., and Pierce, N. A., 2000. "An introduction to the adjoint approach to design". Flow, Turbulence and Combustion, 65(3/4), pp. 393-415.

[19] Giles, M. B., Duta, M. C., M-uacute, J.-D., ller, and Pierce, N. A., 2003. "Algorithm developments for discrete adjoint methods". AIAA Journal, 41(2), pp. 198-205.

[20] Papadimitriou, D. I., and Giannakoglou, K. C., 2006. "Compressor blade optimization using a continuous adjoint formulation". In Proceedings of the ASME Turbo Expo 2006, ASME, pp. 13091317.

[21] Lee, B. J., and Liou, M.-S., 2012. "Unsteady adjoint approach for

Copyright @ 92018 by Rolls-Royce Deutschland Ldt. \& Co KG 
design optimization of flapping airfoils". AIAA Journal, 50(11), pp. 2460-2475.

[22] Initial Training Network, 2015. Industrial optimal design using adjoint cfd. See also URL: http://ioda.sems.qmul.ac.uk/.

[23] Gräsel, J., Keskin, A., Swoboda, M., Przewozny, H., and Saxer, A., 2004. "A full parametric model for turbomachinery blade design and optimisation". In 30th Design Automation Conference, Proceedings of the ASME Design Engineering Technical Conferences and Computers and Information in Engineering Conference - 2004, ASME, pp. 907-914.

[24] Vasilopoulos, I., Agarwal, D., Meyer, M., Robinson, T. T., and Armstrong, C. G., September 2016. "Linking parametric cad with adjoint surface sensitivities". In ECCOMAS Congress 2016, M. Papadrakakis, V. Papadopoulos, G. Stefanou, and V. Plevris, eds., Institute of Structural Analysis and Antiseismic Research School of Civil Engineering National Technical University of Athens (NTUA), pp. 3812-3827.

[25] Vasilopoulos, I., Flassig, P., and Meyer, M., 2017. "Cad-based aerodynamic optimization of a compressor stator using conventional and adjoint-driven approaches". In ASME Turbo Expo 2017, ASME, p. V02CT47A004.

[26] Müller, J. D., and Verstraete, T., 2016. 3d optimization of the tu berlin turbolab stator. See also URL:

https://www. caeses.com/wp-content/ uploads/2016/07/TU_Berlin_Stator.pdf .

[27] Keskin, A., 2007. Process integration and automated multiobjective optimization supporting aerodynamic compressor design: Zugl.: Cottbus, Techn. Univ., Diss., 2006. Berichte aus der Luft- und Raumfahrttechnik. Shaker, Aachen.

[28] Dutta, A. K., 2011. An automated multi-objective optimization approach for aerodynamic 3D compressor blade design: Zugl.: Cottbus, Techn. Univ., Diss., 2011. Berichte aus der Luft- und Raumfahrttechnik. Shaker, Aachen.

[29] Gümmer, V., and Wenger, U., 2000. "The impact of sweep and dihedral on axial compressor endwall aerodynamics". In Proceedings of the 8th International Symposium on Transport Phenomena and Dynamics of Rotating Machinery.

[30] Abbott, I. H., and VonDoenhoff, A. E., 1959. Theory of wing sections: Including a summery of airfoil data, unabridged, corr. republ ed. Dover Publ, New York, N.Y.

[31] Piegl, L., and Tiller, W., 1997. The NURBS Book, second edition ed. Monographs in Visual Communication. Springer, Berlin and Heidelberg.

[32] Shahpar, S., and Lapworth, L., 2003. "Padram: Parametric design and rapid meshing system for turbomachinery optimisation". In Proceedings of the ASME Turbo Expo 2003, American Society of Mechanical Engineers, pp. 579-590.

[33] Lapworth, L., 2004. "Hydra-cfd: A framework for collaborative cfd development". In International Conference on Scientific and Engineering Computation (IC-SEC), Singapore, June, Vol. 30.

[34] Shahpar, S., and Caloni, S., 2013. "Aerodynamic optimization of high-pressure turbines for lean-burn combustion system". Journal of Engineering for Gas Turbines and Power, 135(5), p. 055001.

[35] Giles, M. B., 2002. "On the iterative solution of adjoint equations". In Automatic Differentiation of Algorithms, G. Corliss, C. Faure,
A. Griewank, L. Hascoët, and U. Naumann, eds. Springer New York, New York, NY and s.l., pp. 145-151.

[36] Moinier, P., M-uacute, J.-D., ller, and Giles, M. B., 2002. "Edgebased multigrid and preconditioning for hybrid grids". AIAA Journal, 40(10), pp. 1954-1960.

[37] Deutsche Forschungsgesellschaft, 2012. Collaborative research centre 1029: Substantial efficiency increase in gas turbines through direct use of coupled unsteady combustion and flow dynamics.

[38] Banjac, M., Petrovic, M. V., and Wiedermann, A., 2014. "A new loss and deviation model for axial compressor inlet guide vanes". Journal of Turbomachinery, 136(7), p. 071011. 